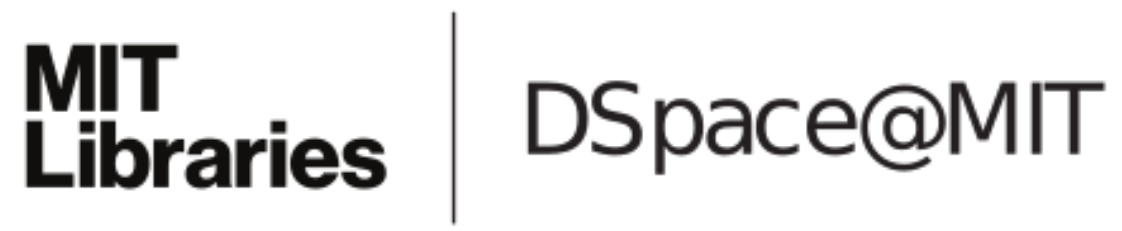

\author{
MIT Open Access Articles
}

\section{Hole-mobility-limiting atomic structures in hydrogenated amorphous silicon}

The MIT Faculty has made this article openly available. Please share how this access benefits you. Your story matters.

Citation: Johlin, Eric, C. B. Simmons, Tonio Buonassisi, and Jeffrey C. Grossman. "Holemobility-limiting atomic structures in hydrogenated amorphous silicon." Phys. Rev. B 90, 104103 (September 2014). (c) 2014 American Physical Society

As Published: http://dx.doi.org/10.1103/PhysRevB.90.104103

Publisher: American Physical Society

Persistent URL: http://hdl.handle.net/1721.1/89217

Version: Final published version: final published article, as it appeared in a journal, conference proceedings, or other formally published context

Terms of Use: Article is made available in accordance with the publisher's policy and may be subject to US copyright law. Please refer to the publisher's site for terms of use. 


\title{
Hole-mobility-limiting atomic structures in hydrogenated amorphous silicon
}

\author{
Eric Johlin, ${ }^{*}$ C. B. Simmons, Tonio Buonassisi, and Jeffrey C. Grossman \\ Massachusetts Institute of Technology, Cambridge, Massachusetts 02139, USA \\ (Received 28 March 2014; revised manuscript received 26 June 2014; published 5 September 2014)
}

\begin{abstract}
Low hole mobility currently limits the efficiency of amorphous silicon photovoltaic devices. We explore three possible phenomena contributing to this low mobility: coordination defects, self-trapping ionization displacement defects, and lattice expansion allowing for hole wave-function delocalization. Through a confluence of experimental and first-principles investigations, we demonstrate the fluidity of the relative prevalence of these defects as film stress and hydrogen content are modified, and that the mobility of a film is governed by an interplay between various defect types.
\end{abstract}

DOI: 10.1103/PhysRevB.90.104103

\section{INTRODUCTION}

In recent years, the rising demand for both low-cost photovoltaic (PV) devices as well as highly efficient heterojunction $\mathrm{PV}$ cells has fostered a notable expansion of research into hydrogenated amorphous silicon (a-Si:H). While a-Si:H PV devices have lingered in the $\sim 10 \%$ efficiency range for more than a decade, and the low hole mobility has been widely accepted as the main barrier to efficiency improvements [1], there remains much disagreement as to the detailed atomic structures responsible for the deficient mobility.

Several recent studies attempt to identify the dominant (or, more realistically, the range of) structural defects responsible for trapping holes in a-Si:H, with theories ranging from the traditional dangling bond (DB; silicon undercoordination) [2-6] and less prevalent floating bond [7,8] explanations, to larger-scale features such as filamentary structures [9] and voids $[10,11]$, to metastable structures such as hydrogen motion [12] and hole self-trapping defects. Our own previous computational investigation into structures influencing hole trapping in a-Si:H found that a particular self-trapping defect showed the strongest correlation with deep hole traps: it was shown that local rearrangement of the a-Si:H atomic structure under the addition of a hole to the system allowed for more energetically favorable localization of a hole in the proximate region (and was thus referred to as an ionization displacement defect) [13]. Floating bonds, or overcoordinated $\mathrm{Si}$ atoms, showed the second largest correlation in our ensembles, while DBs contributed little to the band-tail trap states. Additional works have experimentally measured hole mobilities in deposited films over ranges of deposition conditions [14-16], and modeled the densities of band-tail states implied from these measurements [17-21]. Studies have also sought to measure densities of coordination defects experimentally, namely through electron paramagnetic resonance (EPR), although recent work has shown that such results are challenging to interpret because of the importance of the surrounding geometry on the measurement of the coordination defect [22]. However, despite this abundance of work, a detailed understanding of the atomic structure of hole trapping mechanisms over a range of experimental deposition conditions (necessary to satisfactorily sample the large configuration space possible for a-Si:H) is still lacking.

*johlin@alum.mit.edu
PACS number(s): 61.43.Dq, 71.23.Cq, 71.55.Jv, 72.20.Ee

In this work we aim to connect atomic structure to observed mobility through a series of experiments, leveraging computational simulations to correlate atomic-level phenomena with empirical mobility trends, providing insight into the shifting regimes of prevalent defects. The work is conducted in three main thrusts: First, through experimental measurements of a-Si:H hole mobility over a wide range of deposition conditions, we correlate the mobility with independent measurements of film intrinsic stress, and hydrogen concentration and bonding configuration. We show that the peak mobility values occur at moderately compressive values of stress, away from the extrema of either hydrogen bonding concentration. Second, by extending our previous computational study of structural defects in a-Si:H to include variations in both biaxial stress and hydrogen concentration, we are able to hypothesize on the sources of defects in the various experimental conditions of the material, as well as design experiments to validate these theories. Finally, we deconvolute the stress and hydrogen contents in our as-deposited films and thereby provide experimental evidence to corroborate our computational theories as to the dominant defects and their sources in the continuum of experimental samples produced.

\section{MATERIALS AND METHODS}

\section{A. Experimental sample creation}

All experimental samples are produced via plasmaenhanced chemical vapor deposition (PECVD), in an $n-i-p$ configuration, on degenerately phosphorus-doped $\langle 100\rangle$ crystalline silicon substrates as the rear contact, with nominal layer thicknesses of 25,1000 , and $35 \mathrm{~nm}$, respectively. Intrinsic layer microstructures are varied by changes in deposition chamber pressure. All samples are confirmed to be purely amorphous via micro-Raman spectroscopy, and all layer thicknesses are verified using cross-sectional SEM measurements. Further details of the deposition conditions can be found in the Supplemental Material [23].

\section{B. Experimental characterization}

Hole mobilities are obtained using a custom-built timeof-flight (ToF) transient photocurrent measurement apparatus. The setup consists of an Ekspla NT342B tunable laser system, operating at a $400 \mathrm{~nm}$ wavelength to achieve excitation of carriers confined to the front surface of the device; a pulsed 
voltage source with amplitude $V_{a}$ to generate a drift field; and an Agilent 54855A oscilloscope to measure photocurrent transients. The operating procedure is in accord with Refs. [24-26]. Each device is measured at four or more values of $V_{a}(1-10 \mathrm{~V})$, which is essential to accurately measure mobility, as it is observed both here and elsewhere [27] that ToF mobilities from single-voltage measurements are dependent on the applied bias. Hydrogen content measurements are performed by integrating Gaussian functions fit to the 2000 (Si-H) and 2100 (silicon polyhydride/voids) $\mathrm{cm}^{-1}$ absorption peaks, measured by Fourier transform infrared (FTIR) spectroscopy, following the methods in Ref. [28]. Film stress is calculated via the change in substrate curvature before and after a-Si:H film deposition, using Stoneys formula [29]. The measurements represent the total film stress (intrinsic stress plus thermal stress), although thermal stresses in these films are quite small (likely between 7 and $14 \mathrm{MPa}$ [30]), due to both the similar coefficients of thermal expansion between a-Si:H and the c-Si substrate, as well as the fairly low deposition temperatures $\left(200{ }^{\circ} \mathrm{C}\right)$. Additional details of the measurement systems and analysis can also be found in the Supplemental Material.

\section{Computational sample creation}

The computational results in Ref. [13] show that ionization displacement (ID) defects are predominately responsible for the deep band-tail traps in an a-Si:H ensemble with $\sim 10 \% \mathrm{H}$ and neutral stress. It is known that both the stress and hydrogen content vary depending upon the deposition condition [31]. Here, we capture these variations in our computational models by comparing six different ensembles composed of two hydrogen contents $(\sim 10 \%$ and $\sim 5 \%)$ and three stress states $(-1,0,+1 \mathrm{GPa})$, and we show that these changes in the film properties correspond with a modification in the prevalence and impact of defect types listed above.

Computational samples are constructed by first generating $216 \mathrm{Si}$ plus 10 or $20 \mathrm{H}$ atom structures using a modified Wooten-Winer-Weaire (WWW) [32] process, then relaxing the structures and computing the total energies in the density functional theory (DFT) package SIESTA [33]. The procedures are identical to those discussed and tested in our previous work [13], with the only modifications being the targeting of specific stress states, and an additional ensemble created with a lower, $\sim 5 \%$ hydrogen concentration. Hole trap depth (HTD) analysis also proceeded identically to the above-mentioned work, investigating ensembles of 1200 a-Si:H structures, allowing full relaxations both before and after introduction of the hole into the system, converging these structures to ensure the hole-induced relaxations are fully reversible, and taking the energy difference between these structures as the ionization potential of the structure. This energy is subtracted from the peak of the ionization potential distribution, giving the HTD of a specific structure in the ensemble. After being created, these structures are analyzed to determine the level of presence of various defects. These factors are then used to filter the ensembles to create the conditional probability density plots, allowing for the examination of the influence of a single feature on the full trap distribution. This process is depicted in Fig. 1.

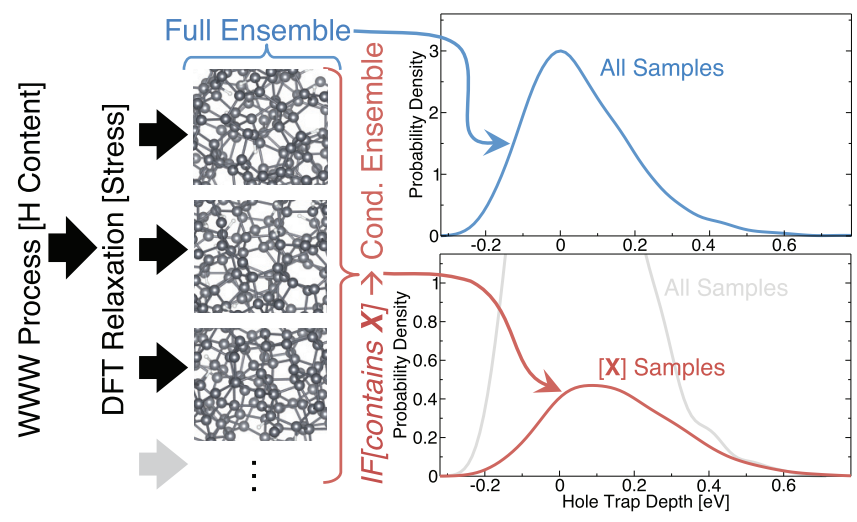

FIG. 1. (Color online) Schematic representation of computational sample creation and analysis process.

Finally, it is important to note that we choose sample properties of stress and hydrogen content to be on the scale of those experimental samples observed, but toward more extreme values to explore trends within a more reasonable sampling size. While modeling conditions identical to physical films is possible, the size of ensembles needed to make unambiguous, statistically significant comparisons between similar films would become computationally prohibitive.

\section{RESULTS AND DISCUSSION}

\section{A. Experimental mobility}

While our films are produced via variations in deposition pressure, we present our experimental trends with respect to the measured film stress, as this provides a coordinate that is both indicative of the physical properties of the material (as opposed to the deposition apparatus), as well as easily, reliably, and accurately measurable. As shown in Fig. 2, the mobilities of our a-Si:H films span an order of magnitude, peaking with a mobility of $\sim 0.01 \mathrm{~cm}^{2} / \mathrm{V} \mathrm{s}$ (similar to that observed in Ref. [16]) at the intermediate compressive stress of approximately $-50 \mathrm{MPa}$, and declining rapidly on either side. We also observe that the silicon monohydride $(\mathrm{Si}-\mathrm{H})$ concentration is monotonically increasing as compressive stress increases, and that the hydrogenated void content starts to increase above its detection limit at stresses $>-100 \mathrm{MPa}$. Other phenomena notable in this plot include that the mobility declines more rapidly in the tensile stress regime than the compressive; that the highest mobility occurs at a nonzero hydrogenated-void concentration; and that the mobility peak appears less stable (larger error bars, and more deviation between samples) than away from said peak. The remainder of this paper is largely devoted to understanding the origins of these phenomena, investigating the interplay between three main defects: floating bonds (FBs), ionization displacement (ID) defects, and lattice expansion (LE) allowing favorable hole delocalization.

\section{B. Computational hole traps}

In Fig. 3, we explore the nature of the extended tails in the distributions of hole trap depths of samples within our six computational ensembles. Specifically, the positive-depth band tails here are of critical importance to hole transport 


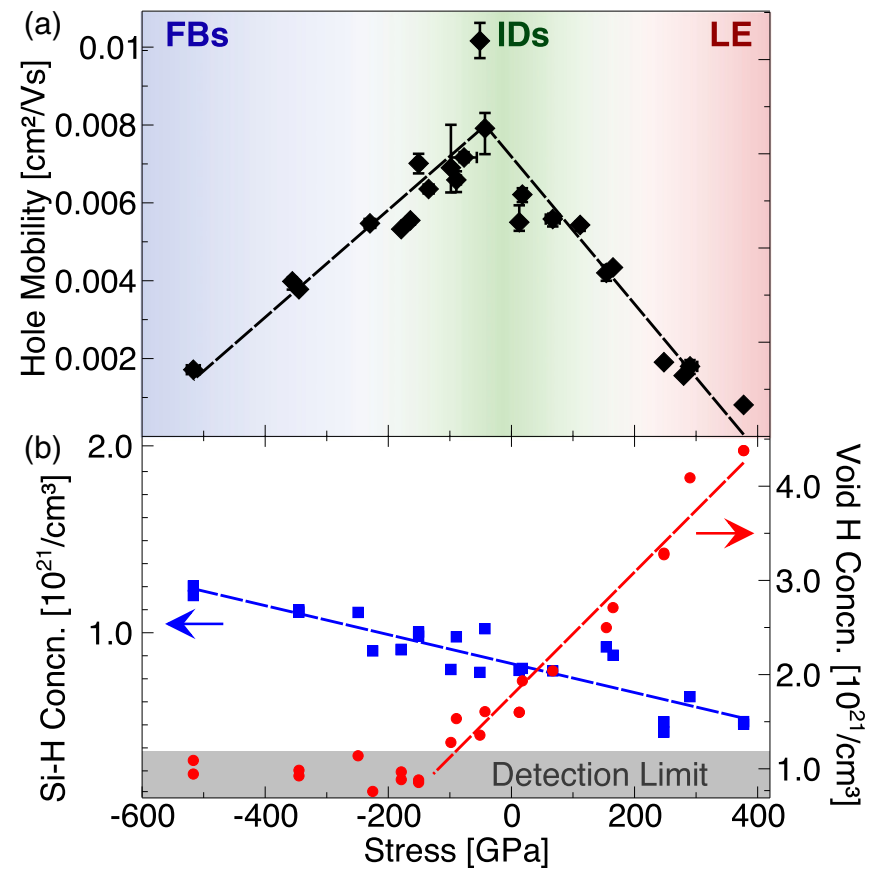

FIG. 2. (Color online) Mobility (a), and film hydrogen content (b) separated into voids (red), and $\mathrm{Si}-\mathrm{H}$ bonded $\mathrm{H}$ (blue), as a function of film stress. Top labels and color shadings indicate the increased defect concentrations in three regions: floating bonds, ionization defects, and lattice expansion. Dashed lines represent linear regressions.

as hole trap time is exponentially dependent on trap depth [21]. In Fig. 3(a), we examine the probabilities (computed using a single-sided two-sample Kolmogorov-Smirnov test) of the positive-depth traps in the ensemble of a given row being smaller than that in a corresponding column. The red-to-blue coloration denotes the low-to-high probability continuum. We observe that the lower hydrogen ensemble has nearly ubiquitously lower trap values than the higher hydrogen states, evident in the $>0.9$ probabilities in the lower-right quadrant of the table. This indicates that at a fixed stress, decreasing hydrogen content corresponds to decreased band-tail HTDs. Within a given hydrogen content, however, we see no ensembles that are clearly $(p>0.9)$ smaller than their counterparts. To understand the more complex behavior of the band tails under stress application, as well as gain insight into the causes of the increasing tails with increasing hydrogen, we decompose the distributions to investigate the specific trap levels and prominence of FBs, DBs [Fig. 3(b)], and IDs [Fig. 3(c)] within our ensembles.

\section{Coordination defects}

In Fig. 3(b), we investigate the influence of structures containing one or more floating or dangling bonds in our ensembles, with bonded silicon defined as atoms with a separation of less than $2.75 \AA$ [13]. We plot density functions of the probability of finding hole trap depths in the ensemble when filtered by said conditions (e.g., contains FBs). This allows us to ascertain the prevalence and severity of the FBs and DBs on the hole trapping in the full ensemble (gray).

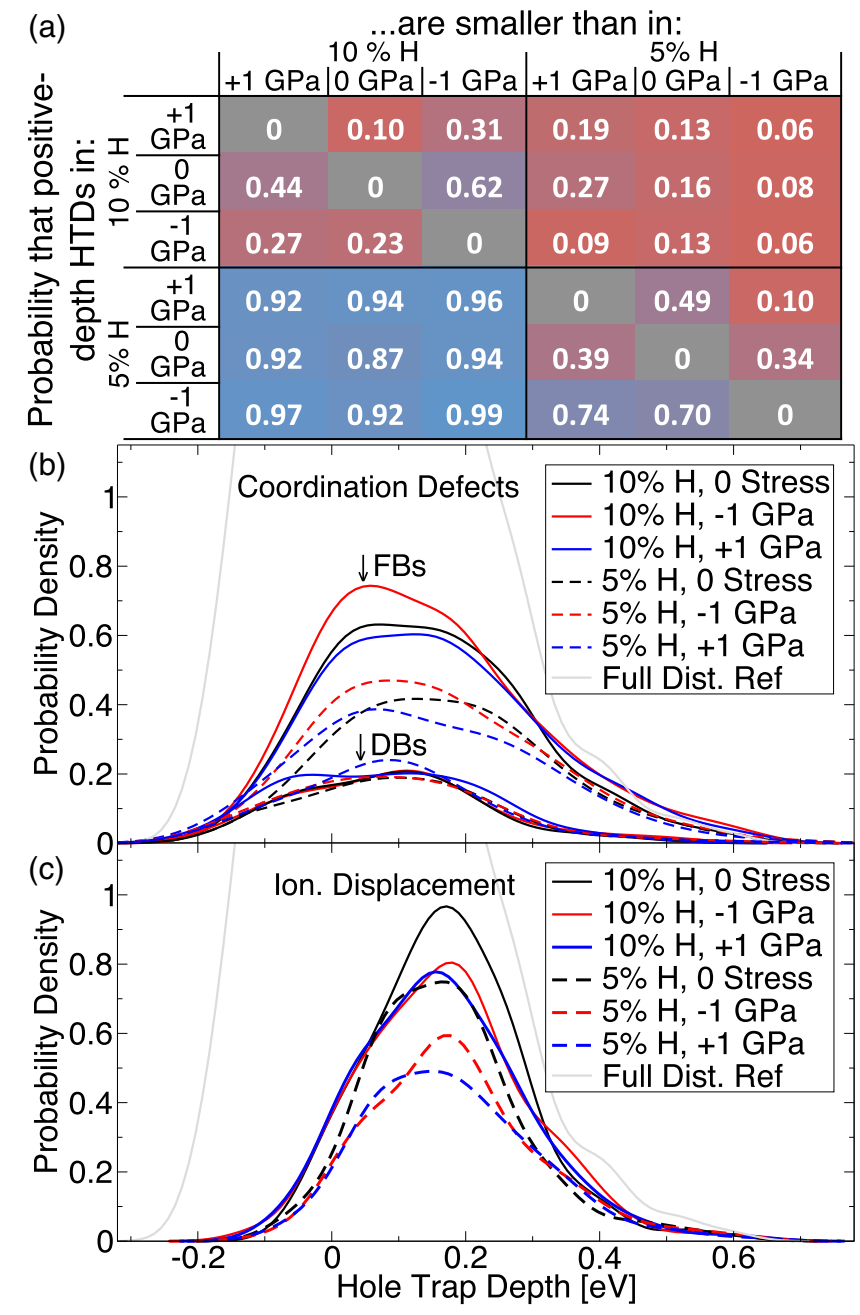

FIG. 3. (Color online) (a) Probabilities that positive HTD tails in the row ensembles are smaller than those in the columns. (b),(c) Conditional probability density distributions of the hole trap depths of computational samples containing (b) FBs and DBs, and (c) IDs. Light gray lines reference full HTD distributions $(10 \% \mathrm{H}$, no stress).

Moving from $10 \%[\mathrm{H}]$ to $5 \%[\mathrm{H}]$ (solid to dashed curves) reduces the density of FBs across the entire HTD distribution, while the application of compressive stress (red) increases the density of FBs relative to the unstressed (black) ensembles, and tensile stress (blue) reduces said density. These trends are easily understood: decreasing the hydrogen in the structure limits the number of bonds needed to be satisfied, and thus decreases the propensity toward FB creation in the structure, while compressing the material decreases bond distances, making FBs more likely. DBs were observed to universally contribute less substantially to the band tails within the studied ensembles, in agreement with previous results $[13,22]$.

\section{Ionization displacement defects}

In Fig. 3(c), we observe that ID defects increase with both increasing $\mathrm{H}$ concentration and decreasing the magnitude of stress in a sample. Here we define IDs as those samples containing an atomic displacement greater than $0.11 \AA$ under the charge removal. We see that in both hydrogen content 


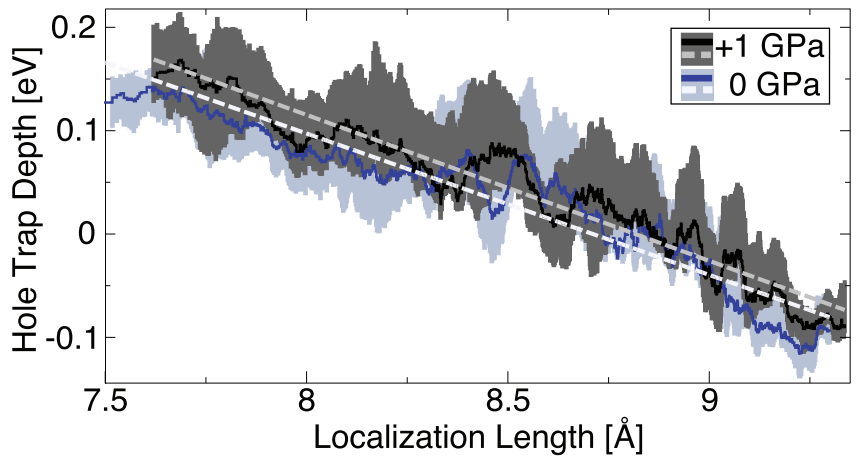

FIG. 4. (Color online) $10 \%$ hydrogen ensemble HTD distributions represented by the peaks (solid lines) and first quartiles (shaded regions) of the probability density, plotted continuously against localization length of the hole wave function. Dashed lines represent linear regressions to the peak values.

ensembles the application of stress reduces the incidence of moderately high HTD ID defects (HTD $>0.1 \mathrm{eV}$ ), but also that decreasing the hydrogen in the samples causes the expression of fewer IDs. We believe that this is due to the less-constrained $\mathrm{Si}-\mathrm{H}$ bonds (hydrogen is usually bonded to only one, or at most two silicon atoms [34]) allowing a higher degree of bond rearrangement under the added potential of a hole being introduced into the system, and thus allowing stronger hole trapping. In the tensile stress regime, it is interesting to note that the decrease in occurrence of IDs and FBs does not explain the overall increased tails in the full hole trap distributions (seen in the generally higher values in the positive stress columns of Fig. 3(a), nor the correspondingly decreased experimental mobility [Fig. 2(a)]. To understand these features we look beyond atomic defects, and investigate the behavior of the hole wave function.

\section{Lattice Expansion}

In Fig. 4 we plot the hole trap depth distribution as a function of the localization length of the hole wave function in our samples, calculated using the methods set forward by Silvestrelli [35] and Resta and Sorella [36]. We observe that the lattice expansion caused by tensile stress allows delocalization of the hole wave function around potential trapping structures, lowering the kinetic energy penalty of a highly localized wave function, thereby yielding deeper trap depths. Considering the mechanism by which IDs trap holes (increasing localization lengths in a given area, causing stronger trapping), it is fitting that by expanding the a-Si:H lattice, tensile stress is essentially leading to the same effect as the IDs in unstressed samples. We see that while both stress states express strong correlations between hole trapping and the localization of the hole, the tensile ensemble shows (1) longer localization lengths than the unstressed ensemble, indicating that tensile stress is indeed allowing delocalization; and (2) that the hole trap depth becomes more dependent on the localization length when tensile stress is applied, seen through the higher slope $(4.4 \mathrm{meV} / \AA)$, confirmed to $99 \%$ confidence, as well as the +1 GPa peak (black) remaining nearly uniformly above the unstressed ensemble (blue). These effects, combined with the rapid increase of hydrogenated voids in our experimental samples, begin to explain why we obtain a sharp decline in hole mobility of our films with increasing tensile stress, as well as agreeing with previous experiments measuring decreased hole mobility under applied stress in polycrystalline films [37].

\section{Density of states}

We next examine how coordination and ionization defects influence the electronic density of states (DOS) of the ensembles. In Fig. 5 we compare the full ensemble average DOS (black) to that of the presence and removal of the three examined defect states: dangling bonds, floating bonds, and ionization displacement. In Fig. 5(a), we note that the DOS of the DB defects increase the density of states mid-gap considerably, but more substantially toward the conduction band states than the valence band, as visible through the arrow connecting the minima of the DOS. Furthermore, the low prevalence of these defects relative to the other two in our ensembles limits their influence on the sample hole trapping, as visible by the minor change in the DOS of the full ensemble with all DBs removed (blue).

In Fig. 5(b), we see that FB defects have a similar influence, although with more equal distribution of defect states onto the valence and conduction bands, and also that the defect presents
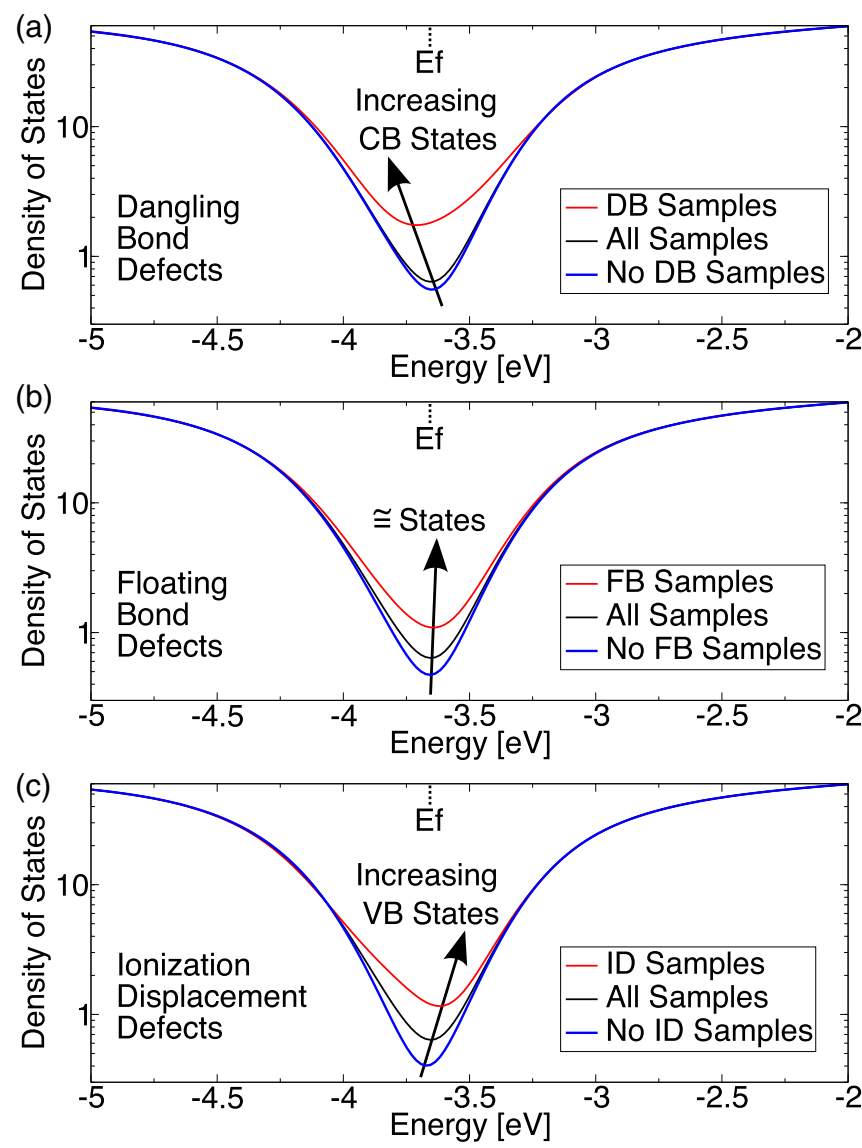

FIG. 5. (Color online) Semilog plots of the electronic densities of states in the $10 \%$ hydrogen, neutral stress ensemble, shown for the positively charged structures. Indicators display relative increases in conduction band (CB) and valence band (VB) state densities, and the bulk Fermi energy (Ef). 
a greater influence on the total ensemble DOS, again visible through the difference between the full ensemble (black) and that with the FB defect-containing samples removed (blue).

Finally, in Fig. 5(c) we observe that ID defects have the most significant influence on the valence band DOS, fitting with our previous observations assigning these defects as the most highly correlated to significant hole trap depths. We also see the removal of these defects as having the most significant influence on the full distribution (difference between blue and black lines) of the three defects.

It should be noted that while the DOS here represent those calculated via generalized gradient approximation (GGA) DFT, which introduces systematic error in the band positions (most notably through the gap underestimation), these errors have been studied in depth and it is believed that, while the absolute positions of the bands contain inaccuracies, the relative behavior within a system should remain accurate $[13,38,39]$. Furthermore, we see the same trends for the uncharged samples, and little difference between the DOS for the six ensembles investigated here (shown in the Supplemental Material).

\section{Connection between experimental mobility and computational hole traps}

Through the confluence of the results presented here, we can establish a better understanding of the mobility trends in Fig. 2. In the compressive stress region, the sharp decline in mobility is attributable to two factors: the high compressive stress levels increase the density of floating bonds substantially, and while the compressive stress also somewhat limits the occurrence of IDs, the concomitant increase in $\mathrm{Si}-\mathrm{H}$ concentration not only counteracts this by increasing IDs, but also further increases the FB density as well. In the tensile regime, while we do see a decline in both the FB and ID concentrations computationally, we also observe the longer localization length of hole wave functions, as well as a stronger dependence between localization and hole trapping in the tensile-stressed samples, indicating that the lattice expansion could be allowing holes to localize over larger regions within the structures and thus become strongly trapped, similar to the filamentary [9] and void $[10,11]$ structural traps explored elsewhere. Finally, these tradeoffs in our structures - between compressive stress limiting the occurrence of IDs, but promoting FBs; as well as a low $\mathrm{Si}-\mathrm{H}$ density being beneficial, but occurring experimentally under otherwise-unfavorable tensile stress and void-rich conditions-explain why we see both a peak mobility at an intermediate stress condition, and why the repeatability of the material quality between devices becomes more unstable as the mobility increases. These findings are summarized through the shading and labeling at the top of Fig. 2(a).

\section{Deconvoluting stress and hydrogen from mobility experimentally}

While these results elucidate atomic configurations potentially limiting the mobility in the various stress regimes of our samples, it is important that we take steps to experimentally vet our explanations. In support of this, we perform an additional experiment with the objective of deconvoluting the total stress in the a-Si:H films from the hydrogen content (and bonding

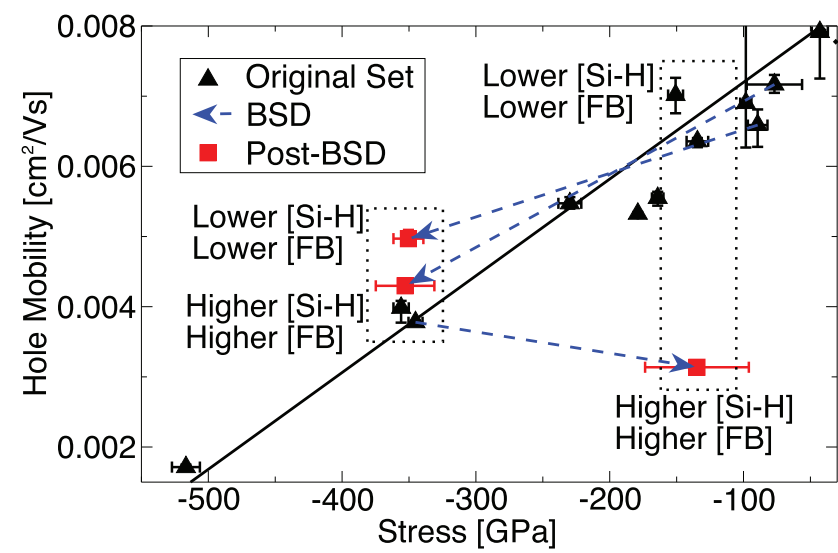

FIG. 6. (Color online) ToF-measured hole mobility of films before (black) and after back-side depositions (BSD; red). Blue dashed lines indicate the movement of samples from the BSD. Dotted boxes indicate isobaric comparisons of differing hydrogen concentrations.

configurations), and determining the independent influences on the film hole mobility. Experimentally, the film stress is modified by depositing an additional amorphous silicon film on the back side of the device substrates, which modifies the curvature of the substrates and therefore the stress in the device films without affecting their hydrogen content. We confirmed that the deposition process had no influence on the mobilities of the front-side films when the stress was not changed, and that the stress modification of the films had no measurable change in the hydrogen content of either bonding configuration in the samples (see Supplemental Material).

The key results are displayed in Fig. 6, confirming that lower $[\mathrm{H}]$ corresponds to improved mobilities. Here we focus on the compressive stress region of the trend in Fig. 2, as both the high prevalence of larger voids makes the tensile region less computationally accessible, and the low mobility of the region makes it less experimentally interesting. We recall from our computational results in Fig. 3(a) that while comparisons between hydrogen concentrations yield statistically relevant results for changes in HTDs, comparisons within a given hydrogen content (varying stress) were indeterminate, so we focus on samples at a single stress, investigating the trend with changing hydrogen content. The first set of samples in this study originates at low compressive stress conditions (ca. $-80 \mathrm{MPa}$ ), and is shifted to increased compressive stress to match previous depositions ( $-350 \mathrm{MPa}$; red squares). At this constant stress condition, we recall that the stress-modified samples contained a lower $\mathrm{Si}-\mathrm{H}$ density than the unmodified samples immediately below them on the plot. We can thus see that at a constant stress, the samples with the lower $\mathrm{Si}-\mathrm{H}$ concentration do exhibit a significantly higher mobility than those with the higher Si-H density. Additionally, by applying the opposite stress movement-taking one of the high compressive stress samples $(-350 \mathrm{MPa})$ and reducing the stress to a more moderate condition $(-135 \mathrm{MPa})$ - we see that this sample is of a lower mobility than those containing a lower $\mathrm{Si}-\mathrm{H}$ concentration. Although direct confirmation of the atomic defects dominating the hole trapping in our samples is experimentally untenable, these experiments do 
provide agreement with our computationally based results, supporting our postulate that the mobility-limit defects are not a single structure, but an interplay between FBs, IDs, as well as potentially further undiscovered defects. We should also note that while lower hydrogen content proved beneficial here, extreme reduction of this property could lead to a new regime in which other deleterious influences begin to dominate.

\section{CONCLUSIONS}

While the results discussed here do not attempt to provide an exhaustive description of the nanostructural hole traps possible in a-Si:H, we believe that they represent an important step in understanding the changing interplay between atomic-level defects in amorphous silicon devices. Furthermore, the presence of multiple defects, controlled by interdependent material properties (e.g., stress, hydrogen content) provides evidence for why despite decades of research, hole mobilities in a-Si:H still fall far below those theoretically possible for free-carrier transport through an amorphous material $[1,19,40]$. Finally, the results and methods presented here can be generalized to the study of other directionally bonded disordered semiconductors, with the evidence of the interdependent nature of defects in these materials solidifying the need for future works to be cognizant of, and adaptable to, this variability.

\section{ACKNOWLEDGMENTS}

We acknowledge and thank M. T. Winkler, D. A. Strubbe, N. Ferralis, and T. R. Kirkpatrick (MIT), and N. A. Tabet (KFUPM) for helpful discussions. The authors would like to thank the King Fahd University of Petroleum and Minerals in Dhahran, Saudi Arabia, for funding the research reported in this paper through the Center for Clean Water and Clean Energy at MIT and KFUPM under Project No. R1-CE-08. The authors also acknowledge the Texas Advanced Computing Center (TACC) at The University of Texas at Austin for providing HPC resources that have contributed to the research results reported within this paper. This work was performed in part at the Center for Nanoscale Systems (CNS), a member of the National Nanotechnology Infrastructure Network (NNIN), which is supported by the National Science Foundation under NSF Grant No. ECS- 0335765.
[1] J. Liang, E. A. Schiff, S. Guha, B. Yan, and J. Yang, Appl. Phys. Lett. 88, 063512 (2006).

[2] Y. Bar-Yam and J. D. Joannopoulos, Phys. Rev. Lett. 56, 2203 (1986).

[3] H. M. Branz and E. A. Schiff, Phys. Rev. B 48, 8667 (1993).

[4] Z. E. Smith and S. Wagner, Phys. Rev. Lett. 59, 688 (1987).

[5] R. A. Street, J. Kakalios, and T. M. Hayes, Phys. Rev. B 34, 3030 (1986).

[6] M. Stutzmann, Philos. Mag. B 60, 531 (1989).

[7] S. T. Pantelides, Phys. Rev. Lett. 57, 2979 (1986).

[8] J. H. Stathis, Phys. Rev. B 40, 1232 (1989).

[9] D. A. Drabold, Y. Li, B. Cai, and M. Zhang, Phys. Rev. B 83, 045201 (2011).

[10] M. Fehr, A. Schnegg, B. Rech, O. Astakhov, F. Finger, R. Bittl, C. Teutloff, and K. Lips, Phys. Rev. Lett. 112, 066403 (2014).

[11] J. Melskens, A. H. M. Smets, M. Schouten, S. W. H. Eijt, H. Schut, and M. Zeman, IEEE J. Photovolt. 3, 65 (2013).

[12] H. M. Branz, Phys. Rev. B 60, 7725 (1999).

[13] E. Johlin, L. K. Wagner, T. Buonassisi, and J. C. Grossman, Phys. Rev. Lett. 110, 146805 (2013).

[14] G. Ganguly and A. Matsuda, Mat. Res. Soc. Symp. Proc. 336, 7 (1994).

[15] G. Ganguly, I. Sakata, and A. Matsuda, J. Non-Cryst. Solids 198, 300 (1996).

[16] B. A. Korevaar, G. J. Adriaenssens, A. H. M. Smets, W. M. M. Kessels, H. Z. Song, M. C. M. van de Sanden, and D. C. Schram, J. Non-Cryst. Solids 266-269, 380 (2000).

[17] M. Brinza and G. J. Adriaenssens, J. Optoelectron. Adv. Mater. 8, 2028 (2006).

[18] C. Main, R. Brüggemann, D. P. Webb, and S. Reynolds, Solid State Communi. 83, 401 (1992).

[19] J. M. Marshall, Rep. Prog. Phys. 46, 1235 (1983).

[20] S. Reynolds, J. Optoelectron. Adv. Mater. 11, 1086 (2009).

[21] E. A. Schiff, Philos. Mag. 89, 2505 (2009).

[22] G. Pfanner, C. Freysoldt, J. Neugebauer, F. Inam, D. Drabold, K. Jarolimek, and M. Zeman, Phys. Rev. B 87, 125308 (2013).
[23] See Supplemental Material at http://link.aps.org/supplemental/ 10.1103/PhysRevB.90.104103 for details of the deposition conditions.

[24] R. Koenenkamp and T. Shimada, Appl. Phys. Commun. 12, 11 (1993).

[25] W. E. Spear, J. Non-Cryst. Solids 60, 1 (1983).

[26] T. Tiedje, C. R. Wronski, B. Abeles, and J. M. Cebulka, Solar Cells 2, 301 (1980).

[27] E. A. Schiff, J. Non-Cryst. Solids 352, 1087 (2006).

[28] A. A. Langford, M. L. Fleet, B. P. Nelson, W. A. Lanford, and N. Maley, Phys. Rev. B 45, 13367 (1992).

[29] L. B. Freund and S. Suresh, Thin Film Materials: Stress, Defect Formation And Surface Evolution (Cambridge University Press, New York, 2003), p. 98.

[30] R. B. Wehrspohn, S. C. Deane, I. D. French, I. Gale, J. Hewett, M. J. Powell, and J. Robertson, J. Appl. Phys. 87, 144 (2000).

[31] E. Johlin, N. Tabet, S. Castro-Galnares, A. Abdallah, M. I. Bertoni, T. Asafa, J. C. Grossman, S. Said, and T. Buonassisi, Phys. Rev. B 85, 075202 (2012).

[32] F. Wooten, K. Winer, and D. Weaire, Phys. Rev. Lett. 54, 1392 (1985).

[33] J. M. Soler, E. Artacho, J. D. Gale, A. Garcia, J. Junquera, P. Ordejón, and D. Sánchez-Portal, J. Phys.: Condens. Matter 14, 2745 (2002).

[34] T. Mueller, E. Johlin, and J. C. Grossman, Phys. Rev. B 89 , 115202 (2014).

[35] P. L. Silvestrelli, Phys. Rev. B 59, 9703 (1999).

[36] R. Resta and S. Sorella, Phys. Rev. Lett. 82, 370 (1999).

[37] P.-C. Kuo, A. Jamshidi-Roudbari, and M. Hatalis, Appl. Phys. Lett. 91, 243507 (2007).

[38] M. Legesse, M. Nolan, and G. Fagas, J. Appl. Phys. 115, 203711 (2014).

[39] S. E. Taylor and F. Bruneval, Phys. Rev. B 84, 075155 (2011).

[40] J. M. Marshall, R. A. Street, M. J. Thompson, and W. B. Jackson, J. Non-Cryst. Solids 97-98, 563 (1987). 